\title{
Stochastic Quantization of the Chern-Simons Theory
}

\author{
L.F.Cugliandolo* \\ Dipartimento di Fisica, Università di Roma, "La Sapienza", \\ I-00185 Roma, Italy \\ G.L.Rossini ${ }^{\dagger}$ and F.A.Schaposnik \\ Departamento de Física, Universidad Nacional de La Plata \\ C.C. 67, 1900 La Plata \\ Argentina
}

\begin{abstract}
We discuss Stochastic Quantization of $d=3$ dimensional non-Abelian Chern-Simons theory. We demonstrate that the introduction of an appropriate regulator in the Langevin equation yields a well-defined equilibrium limit, thus leading to the correct propagator. We also analyze the connection between $d=3$ Chern-Simons and $d=4$ Topological Yang-Mills theories showing the equivalence between the corresponding regularized partition functions. We study the construction of topological invariants and the introduction of a non-trivial kernel as an alternative regularization.
\end{abstract}

\footnotetext{
*Supported by "Commission des Communautés Européennes" (DG XII-G-3)

${ }^{\dagger}$ CONICET-Argentina

${ }^{\ddagger}$ Investigador CIC-Buenos Aires
} 


\section{Introduction}

The construction of Quantum Field Theories which are independent of the space-time metric, Topological Quantum Field Theories (TQFTs), has shown to be a fruitful area of research, with applications both in mathematics and in physics [1]-[8] (See [4] for a complete list of references). In TQFTs there are no local excitations and then the only observables are topological invariants. In this way, Witten has shown how to obtain Donaldson invariants in 4dimensional manifolds [四], Jones polynomials of knot theory [2], etc. From the point of view of physics, TQFTs have been investigated in connection with 2 and 3-dimensional gravity models, 2-dimensional conformal field theories, etc.

Quantization of Topological Field Theories can be envisaged using different approaches. For example, the path-integral method leads to explicit expressions for the partition function and other topological invariants [1] - [6]. Alternatively, one can quantize in the functional Schrödinger representation [3], use canonical quantization for systems with constraints [2, 7] or Stochastic Quantization (SQ) [8]. These two last approaches have revealed, as a bonus, interesting connections between models in different number of spacetime dimensions [9]-[1].

Moreover, the SQ approach [12] to TQFTs has clarified the construction of supersymmetric models by exploiting the connection between Langevin equations and Nicolai maps which trivialize the respective models. This connection, introduced by Parisi-Sourlas [13] and Cecotti-Girardello [14] in low dimensions $(d<4)$ was extended to $d \geq 4$ when quantization of TQFTs was undertaken using the Batalin-Vilkovisky approach [5, 6].

Many aspects related to SQ of TQFTs remain to be fully understood. It is the purpose of the present work to address to some of them. In particular, we carefully discuss the problem of convergence towards equilibrium of the stochastic process associated to non-Abelian Chern-Simons theory. To this end, we introduce an appropriate regulator in the associated Langevin equation showing that the resulting perturbative stochastic diagrams lead to the set of ordinary Feynman diagrams for non-Abelian Chern-Simons theory. We also show that the connection between $d=3$ Chern-Simons model and $d=4$ Topological Yang-Mills (TYM) theory, which was originally established

[9]-[11] without taking care of the convergence problem still holds when a regulating scheme is taken into account. 
To more precisely understand the problems to be discussed, let us briefly review in this Introduction the basic features arising in the quantization of TQFTs. Firstly, since the action $S[\phi]$ for fields $\phi$ in TQFTs does not involve at all the metric of the space-time manifold, several problems regarding quantization are posed. Within the path-integral approach, the metric could enter through BRST terms needed to fix its large (topological) symmetry. One should then be sure that the topological character of the theory is not spoiled by quantization. In fact consider the "quantum action" $S_{Q}[\phi]$ appearing in the path-integral defining the partition function:

$$
S_{Q}\left[\Phi, g_{\mu \nu}\right]=S[\phi]+\left\{Q, W\left[\Phi, g_{\mu \nu}\right]\right\} .
$$

Here $\{Q, W\}$ stands for the BRST commutator of $\mathrm{W}$, a functional of the whole field content $\Phi$ ( $\Phi$ includes the original fields $\phi$, Lagrange multipliers, ghosts, ghost for ghosts, ...) and also of the metric. It is easy to see that:

$$
<\frac{\delta S_{Q}}{\delta g_{\mu \nu}}>=<\left\{Q, \frac{\delta W}{\delta g_{\mu \nu}}\right\}>=0
$$

due to the vanishing of BRST commutators vacuum expectation values [1] However, in order to show that the partition function is indeed metric independent, one has to specify an invariant measure (being the naive measure $D \Phi$ metric dependent). This can be done by taking as integration variables not the original fields $\Phi$ but appropriate tensorial densities [15]-17]:

$$
\hat{\Phi}^{a} \equiv g^{\frac{\omega_{a}}{2}} \Phi^{a}
$$

(here $\omega_{a}$ is a weight associated to $\Phi^{a}$ and $g=\operatorname{det} g_{\mu \nu}$ ).

The partition function $Z$ for the TQFT is then defined as 16]:

$$
Z=\int D \hat{\Phi} \exp \left(-S_{Q}\left[\hat{\Phi}, g_{\mu \nu}\right]\right)
$$

with $\hat{\Phi}$ and $g_{\mu \nu}$ taken as independent variables. One can now easily show from eqs.(1.2) and (1.3) that the topological character of the model is preserved:

$$
<T_{\mu \nu}>\equiv-\frac{2}{\sqrt{g}} \frac{\delta \log Z}{\delta g_{\mu \nu}}=0 .
$$

The same care regarding integration variables has to be taken in defining the partition function for TQFTs within the stochastic quantization approach 
[11]. There are however new problems in this last approach, associated with TQFTs peculiarities. In particular, being the action $S[\phi]$ metric independent, it remains unchanged when passing from Minkowski to Euclidean space. This poses of course a problem since Stochastic Quantization was originally formulated for real Euclidean actions. The problem of how to handle complex actions has nevertheless been carefully analyzed [18, 19] and it has recently received much attention [20, 21].

In a particularly attractive TQFT, and here we come to a main point in this paper, there is another source of problems conspiring against convergence towards equilibrium within the stochastic approach. Indeed, consider 3-dimensional Chern-Simons (CS) theory. Since the corresponding action is linear in derivatives one should expect non-convergence of the associated stochastic process towards equilibrium. In order to solve this problems, refined treatments for the Langevin equation of a CS theory have been proposed. They basically rest on the introduction of non-trivial kernels [21, 22] (this compares to the treatment of fermionic models) both for Abelian and non-Abelian cases or Maxwell terms [21], for the Abelian case, as regulators. In both cases it has been proven that conventional propagators can be obtained very simply reproducing the standard results for Chern-Simons theories.

As stated above, for non-Abelian CS theory, convergence of the stochastic process has been only discussed by introducing an appropriate kernel [21]. This approach poses several difficulties when studying the connection between $d=3$ CS theory and TYM theory. We shall then follow an alternative regularization approach by extending the method employed in [21] (based on the introduction of a regulating Maxwell term for the Abelian theory) to the non-Abelian case. We shall analyze the convergence of the associated stochastic process towards equilibrium and establish the connection between $d=3$ CS theory and $d=4$ TYM theory.

The paper is organized as follows: in Section 2 we introduce a $\operatorname{tr} F_{i j}^{2}$ regulating term in the Langevin equation and show, up to second order in perturbation theory, that stochastic diagrams reproduce the standard Feynman rules for CS theory. Then, in Section 3 we reanalyze the connection between $d=3 \mathrm{CS}$ and $d=4$ TYM theories and study topological features of the resulting 4-dimensional effective theory. We also discuss in this Section the alternative regularization scheme based on the introduction of a non-trivial kernel. Finally, in Section 4 we present a discussion of our results. 


\section{Regulating the stochastic process}

As we stated in the Introduction, $\mathrm{SQ}$ of $d=3$ Chern-Simons theory faces problems of convergence towards equilibrium when the naïve Langevin equation is used. These problems are rooted in two main features of Topological Field Theories. Firstly, being the corresponding action metric-independent, it remains unchanged when passing from Minkowski to Euclidean space, this leading to a purely imaginary Euclidean action. Secondly, being the CS theory linear in its derivatives, it does not ensure a finite equilibrium limit.

$\mathrm{Wu}$ and $\mathrm{Zhu}$ [21] have discussed how to handle both problems. On the one hand, one can employ the so-called complex Langevin equation approach [18]: no inconsistency then arises due to the presence of the $i$-factor in front of the Euclidean CS action. Concerning the second point, these authors introduce as a regulator a $F_{i j}^{2}$ term when studying the Abelian theory. At least in this last case, one ends up with a stochastic process tending to equilibrium and leading to standard results for propagators once the regulator is switched off.

In this Section we shall discuss this problem of convergence for the nonAbelian Chern-Simons theory within the SQ scheme. To this end we introduce a $\operatorname{tr} F_{i j}^{2}$ term and then analyze the convergence of the stochastic process (in the next Section we shall discuss the alternative method of introducing a kernel in the Langevin equation, as proposed in Refs. 21, 22]).

The Euclidean action for $d=3$ non-Abelian Chern-Simons theory with a $\operatorname{tr} F_{i j}^{2}$ regulating term reads (compare with the topologically massive YangMills theory 23]):

$$
S=S_{C S}+S_{\Lambda}
$$

with:

$$
\begin{gathered}
S_{C S}=-\frac{i \kappa}{8 \pi} \operatorname{tr} \int_{M_{3}} d^{3} x \epsilon_{i j k}\left(A_{i} \partial_{j} A_{k}+\frac{2}{3} e A_{i} A_{j} A_{k}\right) \\
S_{\Lambda}=\frac{1}{4 \Lambda^{2}} \operatorname{tr} \int_{M_{3}} d^{3} x F_{i j}^{2}
\end{gathered}
$$

Here $A_{i}(i=1,2,3)$ takes values in the Lie algebra of the gauge group $G$, with generators $t^{a}\left(\operatorname{tr} t^{a} t^{b}=\delta^{a b}\right), \kappa=k e^{2}$ with $k \in Z$, and $M_{3}$ is the 3-dimensional compact manifold. In eq.(2.3), $\Lambda$ is a regulator which will be set to infinity at the end of the computations. This type of regulating Yang-Mills term has 
already been used [24] to study renormalization of the non-Abelian ChernSimons theory. It is important to stress that for finite $\Lambda$ the model becomes metric-dependent. Consequently it is important to determine whether the resulting effective action depends or not on the metric at the quantum level (i.e. whether the quantized theory mantains diffeomorphism invariance, a symmetry of the original Chern-Simons theory). In fact, Chen et al. 24 have argued that diffeomorphism anomaly is indeed absent and our results confirm this fact.

We take as Langevin equation associated with action (2.1) the following one:

$$
\partial_{t} A_{i}(x, t)=-\frac{\delta S}{\delta A_{i}}(x, t)+\eta_{i}(x, t)
$$

where $t$ is the stochastic time $(t \in I \equiv[0, T])$ and $\eta_{i}$ is a gaussian noise taking values in the Lie algebra of the group G. A drift term of the form $D_{i} \Omega$ ( with $D_{i}$ the covariant derivative and $\Omega$ an arbitrary function) can be introduced as a way of (stochastic) gauge fixing 25. Moreover, in the analysis of the connection between $d=3$ Chern-Simons and $d=4$ TYM theories, drift terms naturally appear when one derives Langevin equations by imposing commutativity between BRST transformations and stochastic time evolution [10]. Also, the drift term provides in this context a natural way for introducing an $A_{0}$ component for the gauge field in the route towards the effective 4-dimensional theory. We shall come back to this point in the next Section.

In the SQ approach [12, vacuum expectation values (v.e.v.) are computed as the limit -for stochastic time going to infinity- of stochastic expectation values:

$$
\lim _{T \rightarrow \infty}<F\left[A_{\eta}\right]>_{\eta}=<F[A]>
$$

Here $A_{\eta}$ denotes the solution of Langevin equation giving $\mathrm{A}$ as a functional of the noise; the r.h.s. of eq.(2.5) is the v.e.v. of the functional $F[A]$ for the quantum field theory defined from action (2.1). Concerning stochastic expectation values $<>_{\eta}$, they are computed from the noise correlation functions:

$$
\begin{aligned}
<\eta_{i}^{a}>_{\eta} & =0 \\
<\eta_{i}^{a}\left(x_{1}, t_{1}\right) \eta_{j}^{b}\left(x_{2}, t_{2}\right)>_{\eta} & =2 \delta^{a b} \delta_{i j} \delta^{(3)}\left(x_{1}-x_{2}\right) \delta\left(t_{1}-t_{2}\right) .
\end{aligned}
$$




\begin{tabular}{c|c|c}
\hline \hline DIAGRAM & NOTATION & FORMULA \\
\hline & $e V_{i j k}^{a b c}\left(k, k_{1}, k_{2}\right)$ & $\begin{array}{c}-\frac{i}{2} e f^{a b c}\left[\frac{\kappa}{4 \pi} \epsilon_{i j k}+\frac{1}{\Lambda^{2}}\left(\left(k-k_{1}\right)_{k} \delta_{i j}+\right.\right. \\
\left.\left.+\left(k_{1}-k_{2}\right)_{i} \delta_{j k}+\left(k_{2}-k\right)_{j} \delta_{i k}\right)\right]\end{array}$ \\
\hline & $e^{2} W_{i j k l}^{a b c d}$ & $\begin{array}{c}-\frac{1}{6} e^{2}\left[f^{a b e} f^{c d e}\left(\delta_{i k} \delta_{j l}-\delta_{i l} \delta j k\right)+\right. \\
+f^{a c e} f^{b d e}\left(\delta_{i j} \delta_{k l}-\delta_{i l} \delta j k\right)+ \\
\left.+f^{a d e} f^{c b e}\left(\delta_{i k} \delta_{j l}-\delta_{i j} \delta k l\right)\right]\end{array}$ \\
\hline
\end{tabular}

Table 1: Diagrams for stochastic vertices.

Using the explicit form (2.1)-(2.3) for $S$, Langevin equation in momentum space becomes:

$$
\dot{A}_{i}^{a}=-\frac{1}{\Lambda^{2}} k^{2} P_{i j}(k) A_{j}^{a}+\frac{\kappa}{4 \pi} \epsilon_{i j l} k_{l} A_{j}^{a}+Y_{i}^{a}(k, t),
$$

where $\dot{A}_{i}^{a}=\partial A_{i}^{a} / \partial t$ and $P_{i j}(k)$ and $Y_{i}^{a}(k, t)$ are defined as:

$$
P_{i j}(k)=\delta_{i j}-\frac{k_{i} k_{j}}{k^{2}}
$$

$$
\begin{aligned}
& Y_{i}^{a}(k, t)=\eta_{i}^{a}(k, t)+ \\
& \quad+\frac{e}{(2 \pi)^{3 / 2}} \int d^{3} p d^{3} q \delta(k-p-q) V_{i j k}^{a b c}(k,-p,-q) A_{j}^{b}(p, t) A_{k}^{c}(q, t)+ \\
& \quad+\frac{e^{2}}{(2 \pi)^{3}} \int d^{3} p d^{3} q d^{3} r \delta(k-p-q-r) W_{i j k l}^{a b c d} A_{j}^{b}(p, t) A_{k}^{c}(q, t) A_{l}^{d}(r, t) .
\end{aligned}
$$

In the last equation $V_{i j k}^{a b c}$ and $W_{i j k l}^{a b c d}$ are, respectively, the three-point and four-point vertex factors (listed in Table 1 together with the corresponding diagrams). Notice that the three-point vertex factor is just $\frac{1}{2}$ of the corresponding one in the conventional Feynman rule, while the four-point vertex factor is $\frac{1}{6}$ of the corresponding conventional one.

The Langevin equation can be solved perturbatively, and for this purpose it is convenient to write it as an integral equation. Using $A_{i}^{a}(k, 0)=0$ as 
Figure 1: Perturbative expansion of $A_{i}^{a}(k, t)$.

initial condition, one readily obtains

$$
A_{i}^{a}(k, t)=\int_{0}^{\infty} d t^{\prime} G_{i j}^{a b}\left(k, t-t^{\prime}\right) Y_{j}^{b}\left(k, t^{\prime}\right),
$$

where $G_{i j}^{a b}\left(k, t-t^{\prime}\right)$ is the zeroth order (causal) Green function associated with eq.(2.7):

$$
\delta^{a b}\left(\delta_{i j} \frac{\partial}{\partial t}+\frac{k^{2}}{\Lambda^{2}} P_{i j}(k)-\frac{\kappa}{4 \pi} \epsilon_{i j l} k_{l}\right) G_{j m}^{b c}\left(k, t-t^{\prime}\right)=\delta^{a c} \delta_{i m} \delta\left(t-t^{\prime}\right),
$$

which yields

$$
\begin{array}{r}
G_{i j}^{a b}\left(k, t-t^{\prime}\right)=\theta\left(t-t^{\prime}\right) \delta^{a b}\left\{\left[P_{i j}(k) \cos \left(\frac{\kappa}{4 \pi} k\left(t-t^{\prime}\right)\right)+\right.\right. \\
\left.\left.+\frac{\epsilon_{i j l} k_{l}}{k} \sin \left(\frac{\kappa}{4 \pi} k\left(t-t^{\prime}\right)\right)\right] e^{-\frac{1}{\Lambda^{2}} k\left(t-t^{\prime}\right)}+\frac{k_{i} k_{j}}{k^{2}}\right\} .
\end{array}
$$

It is convenient to write eq. $(2.10)$ in a symbolic way as

$$
A=G\left(\eta+e V A A+e^{2} W A A A\right) .
$$

The perturbative expansion of the solution then reads:

$$
\begin{aligned}
A= & G \eta+e G V(G \eta)(G \eta)+e^{2} W(G \eta)(G \eta)(G \eta)+ \\
& +e^{2} G V(G V(G \eta)(G \eta))(G \eta)+e^{2} G V(G \eta)(G V(G \eta)(G \eta))+\cdots
\end{aligned}
$$

which is graphically represented in Figure 1.

In order to compute an $n$-point correlation function $<A_{i_{1}}^{a_{1}}\left(k_{1}, t\right) \cdots$ $A_{i_{n}}^{a_{n}}\left(k_{n}, t\right)>_{\eta}$, one proceeds as follows: for each $A_{i}^{a}(k, t)$ one draws a diagrammatical expansion as that shown in Figure 1, then one takes the average over 
the noise by joining all crosses (representing the noise sources), pair-wise, in all possible ways and finally one sums up the resulting "stochastic diagrams", as implied by the noise correlation functions (see eq.(2.6)).

Notice that every diagram is built up from two kinds of propagators:

a) the un-crossed propagator given by the stochastic Green function (2.12):

$$
\begin{array}{r}
G_{i j}^{a b}\left(k, t-t^{\prime}\right)=\theta\left(t-t^{\prime}\right) \delta^{a b}\left\{\left[P_{i j}(k) \cos \left(m k\left(t-t^{\prime}\right)\right)+\right.\right. \\
\left.\left.+\frac{\epsilon_{i j l} k_{l}}{k} \sin \left(m k\left(t-t^{\prime}\right)\right)\right] e^{-\frac{1}{\Lambda^{2}} k\left(t-t^{\prime}\right)}+\frac{k_{i} k_{j}}{k^{2}}\right\} .
\end{array}
$$

b) the crossed propagator which corresponds to the lowest order term in $<A_{i}^{a}(k, t) A_{j}^{b}\left(-k, t^{\prime}\right)>_{\eta}$ :

$$
\begin{aligned}
& D_{i j}^{a b}\left(k ; t, t^{\prime}\right)=\delta^{a b} 2 t_{\min } \frac{k_{i} k_{j}}{k^{2}}+ \\
& +\delta^{a b} \frac{1}{\frac{k^{2}}{\Lambda^{4}}+m^{2}}\left\{e ^ { - \frac { k ^ { 2 } } { \Lambda ^ { 2 } } | t - t ^ { \prime } | } \left[\left(\frac{1}{\Lambda^{2}} P_{i j}(k)+\frac{m}{k} \frac{\epsilon_{i j l} k_{l}}{k}\right) \cos \left(m k\left|t-t^{\prime}\right|\right)+\right.\right. \\
& \left.\quad+\left(-\frac{m}{k} P_{i j}(k)+\frac{1}{\Lambda^{2}} \frac{\epsilon_{i j l} k_{l}}{k}\right) \sin \left(m k\left|t-t^{\prime}\right|\right)\right]+ \\
& \quad-e^{-\frac{k^{2}}{\Lambda^{2}}\left(t+t^{\prime}\right)}\left[\left(\frac{1}{\Lambda^{2}} P_{i j}(k)+\frac{m}{k} \frac{\epsilon_{i j l} k_{l}}{k}\right) \cos \left(m k\left(t+t^{\prime}\right)\right)+\right. \\
& \left.\left.\quad+\left(-\frac{m}{k} P_{i j}(k)+\frac{1}{\Lambda^{2}} \frac{\epsilon_{i j l} k_{l}}{k}\right) \sin \left(m k\left(t+t^{\prime}\right)\right)\right]\right\}
\end{aligned}
$$

In the last equation $m$ stands for $\kappa / 4 \pi$, usually called the "topological mass" of the model [23], and $t_{\text {min }}$ is the minimum between $t$ and $t^{\prime}$. From the above expressions one recognizes a linearly divergent longitudinal part which is common in the SQ of all gauge theories without gauge fixing. This term can be handled by stochastic gauge fixing [25] (see below).

If not for the presence of two kinds of propagators, each stochastic diagram has the form of an ordinary Feynman diagram. Though the rules for these stochastic propagators are different from the Feynman rules, it has been proven for several models that the sum of all stochastic diagrams with the same topology yields exactly the usual result for the corresponding Feynman diagram. In particular, Namiki et al. [26] have shown that, up to second order, this is the case for pure Yang-Mills theory. In the same spirit we shall 
study the second order corrections to the propagator of the non-Abelian Chern-Simons model (with the $\operatorname{tr} F_{i j}^{2}$ regulator term) and we shall show that its transverse part reproduces the correct field-theoretical propagator.

As stressed above, correlation functions $<A_{i}^{a}\left(k_{1}, t\right) A_{j}^{b}\left(k_{2}, t^{\prime}\right)>$ contain terms which diverge as $t$ and $t^{\prime}$ go to infinity. These divergent terms are characteristic of gauge non-invariant quantities and will cancel out if one constructs gauge-invariant objects like $\left\langle\operatorname{tr} F_{i j}^{2}>\right.$. Consequently we shall only keep in what follows those terms which remain finite as $t$ or $t^{\prime}$ go to infinity. Using (2.14) and averaging over $\eta$ we then get for the correlation function, up to second order:

$$
<A_{i}^{a}(k, t) A_{j}^{b}\left(k^{\prime}, t\right)>=\delta\left(k+k^{\prime}\right) \mathcal{D}_{i j}^{a b}(k, t)
$$

with:

$$
\mathcal{D}_{i j}^{a b}(k, t)=(a)+2(b)+2(c)+2(d)+3(c)+(f),
$$

where $(a),(b), \ldots,(f)$ stand for the contributions from diagrams shown in Figure 2. As an example, diagram $(b)$ represents the following contribution:

$$
\begin{aligned}
2(b) & =\frac{2 e^{2}}{(2 \pi)^{3}} \int d^{3} k_{1} d^{3} k_{2} \delta\left(k-k_{1}-k_{2}\right) V_{k l m}^{c d e}\left(k,-k_{1},-k_{2}\right) V_{q n p}^{f g h}\left(-k, k_{1}, k_{2}\right) \times \\
& \times \int_{0}^{\infty} d t^{\prime} \int_{0}^{\infty} d t^{\prime \prime} G_{i k}^{a c}\left(k, t-t^{\prime}\right) D_{l n}^{d g}\left(k_{1} ; t^{\prime}, t^{\prime \prime}\right) D_{m p}^{e h}\left(k_{2} ; t^{\prime}, t^{\prime \prime}\right) G_{j q}^{b f}\left(-k, t-t^{\prime \prime}\right) .
\end{aligned}
$$

Here it should be noted that longitudinal components of external lines can be discarded either because they vanish or because they cancel out when computing gauge invariant or gauge covariant contributions. This is carefully shown in Ref.26 for the pure Yang-Mills model and the demonstration trivially applies to the present model. Concerning longitudinal components appearing in internal lines, it has been conjectured by Parisi and $\mathrm{Wu}$ [12 that they arrange themselves to give just those contributions which are conventionally associated with Faddeev-Popov ghost effects. Although a general proof for this conjecture is lacking, it has been explicitely confirmed in several examples [27]. In particular, Namiki et al. [26] have proved that, up to second order, in the case of pure Yang-Mills theory internal lines do arrange to reproduce the ghost contributions. Since the longitudinal part of the propagators of this last theory coincide with those arising in the CS model 
Figure 2: Graphical representation of $\mathcal{D}_{i j}^{a b}(k, t)$.

(eqs.(2.16) and (2.17)), we conclude that also in the present case ghost effects are accounted by longitudinal parts of internal lines and hence we shall only consider in diagrams $(a),(b), \ldots,(f)$ transverse internal lines.

Computation of contributions from different diagrams is standard but tedious. For the sake of brevity we shall not present each one separately. Just as an example, the contribution from diagram (b) takes the form:

$$
\begin{aligned}
2(b)=\frac{e^{2}}{(2 \pi)^{3}} & \int d^{3} k_{1} d^{3} k_{2} \delta\left(k-k_{1}-k_{2}\right) V_{k l m}^{a d e}\left(k,-k_{1},-k_{2}\right) V_{q n p}^{\text {bde }}\left(-k, k_{1}, k_{2}\right) \\
& \times I_{i k l n m p q j}\left(k, k_{1}, k_{2}, m, \Lambda\right),
\end{aligned}
$$

where

$$
I_{i k l n m p q j}\left(k, k_{1}, k_{2}, m, \Lambda\right)=
$$




$$
\begin{aligned}
& =P_{i k}(k) P_{l n}\left(k_{1}\right) P_{m p}\left(k_{2}\right) P_{j q}(k) F^{(1)}+P_{i k}(k) P_{l n}\left(k_{1}\right) P_{m p}\left(k_{2}\right) \frac{\epsilon_{j q v} k_{v}}{k} F^{(2)}+ \\
& +P_{i k}(k) P_{l n}\left(k_{1}\right) \frac{\epsilon_{m p u} k_{2 u}}{k_{2}} P_{j q}(k) F^{(3)}+P_{i k}(k) P_{l n}\left(k_{1}\right) \frac{\epsilon_{m p u} k_{2 u}}{k_{2}} \frac{\epsilon_{j q v} k_{v}}{k} F^{(4)}+ \\
& +P_{i k}(k) \frac{\epsilon_{l n s} k_{1 s}}{k_{1}} P_{m p}\left(k_{2}\right) P_{j q}(k) F^{(5)}+P_{i k}(k) \frac{\epsilon_{l n s} k_{1 s}}{k_{1}} P_{m p}\left(k_{2}\right) \frac{\epsilon_{j q v} k_{v}}{k} F^{(6)}+ \\
& +P_{i k}(k) \frac{\epsilon_{l n s} k_{1 s}}{k_{1}} \frac{\epsilon_{m p u} k_{2 u}}{k_{2}} P_{j q}(k) F^{(7)}+P_{i k}(k) \frac{\epsilon_{l n s} k_{1 s}}{k_{1}} \frac{\epsilon_{m p u} k_{2 u}}{k_{2}} \frac{\epsilon_{j q v} k_{v}}{k} F^{(8)}+ \\
& +\frac{\epsilon_{i k r} k_{r}}{k} P_{l n}\left(k_{1}\right) P_{m p}\left(k_{2}\right) P_{j q}(k) F^{(9)}+\frac{\epsilon_{i k r} k_{r}}{k} P_{l n}\left(k_{1}\right) P_{m p}\left(k_{2}\right) \frac{\epsilon_{j q v} k_{v}}{k} F^{(10)}+ \\
& +\frac{\epsilon_{i k r} k_{r}}{k} P_{l n}\left(k_{1}\right) \frac{\epsilon_{m p u} k_{2 u}}{k_{2}} P_{j q}(k) F^{(11)}+\frac{\epsilon_{i k r} k_{r}}{k} P_{l n}\left(k_{1}\right) \frac{\epsilon_{m p u} k_{2 u}}{k_{2}} \frac{\epsilon_{j q v} k_{v}}{k} F^{(12)}+ \\
& +\frac{\epsilon_{i k r} k_{r}}{k} \frac{\epsilon_{l n s} k_{1 s}}{k_{1}} P_{m p}\left(k_{2}\right) P_{j q}(k) F^{(13)}+\frac{\epsilon_{i k r} k_{r}}{k} \frac{\epsilon_{l n s} k_{1 s}}{k_{1}} P_{m p}\left(k_{2}\right) \frac{\epsilon_{j q v} k_{v}}{k} F^{(14)}+ \\
& +\frac{\epsilon_{i k r} k_{r}}{k} \frac{\epsilon_{l n s} k_{1 s}}{k_{1}} \frac{\epsilon_{m p u} k_{2 u}}{k_{2}} P_{j q}(k) F^{(15)}+\frac{\epsilon_{i k r} k_{r}}{k} \frac{\epsilon_{l n s} k_{1 s}}{k_{1}} \frac{\epsilon_{m p u} k_{2 u}}{k_{2}} \frac{\epsilon_{j q v} k_{v}}{k} F^{(16)}
\end{aligned}
$$

Here $F^{(i)}=F^{(i)}\left(k, k_{1}, k_{2}, m, \Lambda\right)$ are ratios of polynomials in momenta which arise after integrating the fictitious time in the vertices and taking the limit $t \rightarrow \infty$. Contributions from the rest of the diagrams having the same topology as (b) have analogous form. Computations were carried out using REDUCE and the answer is:

$$
\begin{aligned}
& 2(b)+2(c)+2(d)= \\
& 2 \frac{e^{2}}{(2 \pi)^{3}} \int d^{3} k_{1} d^{3} k_{2} \delta\left(k-k_{1}-k_{2}\right) V_{k l m}^{a d e}\left(k,-k_{1},-k_{2}\right) V_{b d e}^{f g h}\left(-k, k_{1}, k_{2}\right) \times \\
& \quad \frac{\left(\frac{1}{\Lambda^{2}} P_{i k}(k)+\frac{m}{k^{2}} \epsilon_{i k r} k_{r}\right)}{\frac{k^{2}}{\Lambda^{4}}+m^{2}} \frac{\left(\frac{1}{\Lambda^{2}} P_{l n}\left(k_{1}\right)+\frac{m}{k_{1}^{2}} \epsilon_{l n s} k_{1 s}\right)}{\frac{k_{1}^{2}}{\Lambda^{4}}+m^{2}} \\
& \quad \frac{\left(\frac{1}{\Lambda^{2}} P_{m p}\left(k_{2}\right)+\frac{m}{k_{2}^{2}} \epsilon_{m p u} k_{2 u}\right)}{\frac{k_{2}^{2}}{\Lambda^{4}}+m^{2}} \frac{\left(\frac{1}{\Lambda^{2}} P_{j q}(k)+\frac{m}{k^{2}} \epsilon_{j q v} k_{v}\right)}{\frac{k^{2}}{\Lambda^{4}}+m^{2}}
\end{aligned}
$$

An analogous calculation for diagrams $(e)$ gives: 


$$
\begin{aligned}
3(e) & =3 \frac{e^{2}}{(2 \pi)^{3}} \int d^{3} k_{1} W_{k l m n}^{a d d b} \times \\
& \frac{\left(\frac{1}{\Lambda^{2}} P_{i k}(k)+\frac{m}{k^{2}} \epsilon_{i k r} k_{r}\right)}{\frac{k^{2}}{\Lambda^{4}}+m^{2}} \frac{\left(\frac{1}{\Lambda^{2}} P_{l m}\left(k_{1}\right)+\frac{m}{k_{1}^{2}} \epsilon_{l m u} k_{u}\right)}{\frac{k_{1}^{2}}{\Lambda^{4}}+m^{2}} \frac{\left(\frac{1}{\Lambda^{2}} P_{j n}(k)+\frac{m}{k^{2}} \epsilon_{j n s} k_{s}\right)}{\frac{k^{2}}{\Lambda^{4}}+m^{2}}
\end{aligned}
$$

Taking into account the relations between stochastic and conventional Feynman vertices, it is now evident that eqs.(2.22) and (2.23) coincide, respectively, with the gluon loop and the tadpole terms in the conventional Landau gauge field theory (see for instance [28]). Finally, diagram $(f)$ vanishes due to antisymmetry of the three-point vertex. Hence we arrive to:

$(a)+2(b)+2(c)+2(d)=$

$3(e)+(f)=$

Here wavy lines represent the standard (bare) propagator for topologically massive gauge theory, i.e. $d=3$ Yang-Mills theory plus a CS term (compare e.g. Ref. 228]):

$$
=\frac{\left(\frac{1}{\Lambda^{2}} P_{i j}(k)+\frac{m}{k^{2}} \epsilon_{i j r} k_{r}\right)}{\frac{k^{2}}{\Lambda^{4}}+m^{2}}
$$

Thus, the r.h.s. in (2.24) and (2.25) reproduce the standard second order result in perturbation theory. Concerning the resulting three-point and four-point vertices, they coincide with the standard vertices for topologically massive gauge theory and they can be read off from those presented in Table 1 (provided we disregard the factors $\frac{1}{2}$ and $\frac{1}{6}$ respectively, appearing in the stochastic scheme). 
We can therefore conclude that, up to second order in perturbation, the Stochastic Quantization scheme for non-Abelian CS model endowed with a regulator term of the form (2.3) leads to the correct gauge field propagator for topologically massive Yang-Mills theory. That is, the introduction of the $\frac{1}{\Lambda^{2}} t r F_{i j}^{2}$ term has lead to a stochastic process which converges towards equilibrium. As we have already stated, diagrams with ghosts lines should be obtained by including the longitudinal parts of $G_{i j}^{a b}$ and $D_{i j}^{a b}$ in the internal lines of each diagram.

If one now takes the limit of $\Lambda^{2} \rightarrow \infty$ to switch off the regulator so as to recover the pure CS gauge theory, one can easily verify that (2.24)-(2.26) agree with the standard (bare) expresions (compare e.g. Ref. 29.).

We have then succeded in obtaining a convergent stochastic process for the non-Abelian CS theory. We can then re-analize the connection between the (regulated) stochastic partition function for CS theory and the BRST partition function for TYM theory. This will be presented in the next Section.

\section{The connection between CS and TYM the- ories}

The connection between $d=3$ non-Abelian CS and $d=4$ TYM theories has been established by $\mathrm{Yu}$ [9] and Baulieu [10] using SQ. Alternatively, Horowitz [7] has discussed the same connection using canonical quantization for systems with constraints.

Concerning the SQ derivation, it consists in proving the equivalence between the stochastic partition function for CS theory in the limit $T \rightarrow \infty$ and the BRST partition function for TYM theory (stochastic time $t$ provides the extra coordinate necessary to pass from $M_{3}$ to a $d=4$ manifold $M_{4}$ ).

The analysis in [9, 10] did not take into account the necessity of working with an invariant measure for the path-integral which defines the partition functions. As mentioned in the Introduction this poses a problem since the naïve measure is in fact metric dependent, and hence the topological character of the quantum theory cannot be simply established. In [11] this problem was studied using the so-called Fujikawa variables. This variables allow to define an invariant measure at the price of working not with the original fields but with appropriate tensorial densities (see eq.(1.3) and (1.4)). The 
outcome is that the connection between CS and TYM partition functions is still valid when the correct integration variables are considered.

Another important point that was not taken into account in the derivations given by [9]-11] based in SQ was that related to the non-convergence of the underlying stochastic process. We have proven in the previous Section that the introduction of a regulator in the Langevin equation yields the correct equilibrium limit for the stochastic process and gives the standard propagators for the non-Abelian CS theory. Once this is achieved, one has to re-examine the question of whether the connection between CS and TYM theories is still valid when the theory is regulated. We address to this point in the first part of this Section and show that the answer is affirmative. Then we also analyze the same problem when a non-trivial kernel (instead of a regulator) is introduced in the Langevin equation, using a slightly different approach.

In order to proceed to the proof of the connection à la Yu-Baulieu, we have to modify Langevin equation (2.4) for the CS model adding a longitudinal drift term. In fact when one derives Langevin equations by imposing commutativity between BRST transformations and stochastic time evolution, a drift term naturally appears [10]. This term can be in fact identified with the one which is usually introduced in order to handle the non-convergence of gauge dependent v.e.v.'s within the SQ approach. In the present context, it provides a natural way for introducing an $A_{0}$ component for the CS gauge field in the route towards the 4-dimensional theory. Indeed, if instead of eq. 2.4) we write a Langevin equation with drift term:

$$
\partial_{t} A_{i}=-\frac{\delta S}{\delta A_{i}}-D_{i} \Omega+\eta_{i}
$$

being $\Omega$ an arbitrary function, we can take:

$$
\Omega(x, t)=-\frac{\kappa}{4 \pi} A_{0}(x, t) .
$$

Here $A_{0}$ will be considered, as announced, the zeroth component of the gauge field $A_{\mu} \equiv\left(A_{i}, A_{0}\right)$, now defined over a four manifold $M_{4}=M_{3} \times I$. We then impose a Langevin equation for $A_{0}$ :

$$
\frac{\partial A_{0}(x, t)}{\partial t}=-\frac{\kappa}{4 \pi} \partial_{i} A_{i}+\eta(x, t)
$$


with $\eta$ a scalar gaussian noise taking values in the Lie algebra of the group G.

In order to write equations (3.1) and (3.3) in a more appropriate way, we introduce $\partial_{0}=\frac{4 \pi}{\kappa} \partial_{t}$. We also redefine $e A_{\mu} \rightarrow A_{\mu}$ and set $e^{2}=\frac{\kappa}{4 \pi}$. Langevin equations take then the form:

$$
F_{0 i}^{+}-\frac{4 \pi}{\kappa \Lambda^{2}} D_{j} F_{j i}=\sqrt{\frac{4 \pi}{\kappa}} \eta_{i}
$$

and

$$
\partial_{\mu} A_{\mu}=\sqrt{\frac{4 \pi}{\kappa}} \eta
$$

Here

$$
F_{\mu \nu}=\partial_{\mu} A_{\nu}-\partial_{\nu} A_{\mu}+i e\left[A_{\mu}, A_{\nu}\right]
$$

and

$$
F_{\mu \nu}^{+} \equiv F_{\mu \nu}+\frac{1}{2} \epsilon_{\mu \nu \alpha \beta} F_{\alpha \beta} .
$$

We are now ready to establish the connection between $d=4$ TYM and $d=3$ CS theory (regulated with the $\operatorname{tr} F_{i j}^{2}$ term) when the regulator is switched off. We start from the stochastic generating functional for the theory associated with equations (3.4) and (3.5):

$$
Z_{\text {Stoch }}^{C S}[\Lambda]=\int D \eta_{i} D \eta \exp \left[-\frac{2 \pi}{\kappa} \operatorname{tr} \int_{M_{4}} d^{4} x\left(\eta_{i}^{2}+\eta^{2}\right)\right]
$$

where $d^{4} x=d^{3} x d x_{0}$ We have used in eq.(3.8) a stochastic gaussian measure defined in terms of the original gaussian noises $\eta_{i}$ and $\eta$ and not in terms of the associated Fujikawa variables [15]

$$
\hat{\eta}_{i}=g^{1 / 12} \eta_{i} \quad \hat{\eta}=g^{1 / 4} \eta
$$

As we stated above, these last ones are the correct variables defining an invariant measure. However, as it was proven in [11], the derivation of the connection between CS and TYM theories follows exactly the same steps using ordinary or Fujikawa variables and hence, for the sake of simplicity, we shall work with the original variables although our proof can be straightforwardly presented in terms of Fujikawa variables. 
We now write $Z_{\text {Stoch }}^{C S}[\Lambda]$ as a path integral over gauge fields $A_{\mu}$ by using

$$
D \eta D \eta_{i}=J D A_{\mu}
$$

with the Jacobian given by

$$
J=\operatorname{det} M_{i \nu}
$$

and

$$
M_{i \nu}=\left(\begin{array}{c}
\frac{\delta \eta}{\delta A_{\nu}} \\
\frac{\delta \eta_{i}}{\delta A_{\nu}}
\end{array}\right)
$$

Ghost fields $\left(b, \chi_{i}\right)$ and $\psi_{\nu}$ (with ghost numbers $(-1,-1)$ and 1 respectively) can be introduced in order to exponentiate $M_{i \nu}$ :

$$
J=\int D b D \chi_{i} D \psi_{\mu} \exp \left[\operatorname{tr} \int_{M_{4}} d^{4} x\left(b, \chi_{i}\right) M_{i \nu} \psi_{\nu}\right]
$$

Of course, the change of variables holds for $J \neq 0$ (which corresponds, in the limit $\Lambda \rightarrow \infty$, to $\operatorname{dim} \mathcal{M}=0$, being $\mathcal{M}$ the instanton moduli space associated with $F_{\mu \nu}^{+}=0$ equation $)$. The case $J=0(\operatorname{dim} \mathcal{M} \neq 0)$ can be easily treated by extending the procedure which we describe below (see Ref. [9] for details).

In order to end up with the complete set of ghosts characterizing BRST invariance of TYM theory we introduce, following [32], $c$-ghosts (associated with ordinary gauge symmetry in TYM) through the equation:

$$
D_{\mu} D_{\mu} c-D_{\mu} \psi_{\mu}=\beta
$$

with $\beta$ a gaussian noise. Inserting the identity

$$
1=\int D \rho D \beta \exp \left[-\operatorname{tr} \int \rho \beta d^{3} x d t\right]
$$

in $Z_{\text {Stoch }}^{C S}$ and changing from $\beta$ to $c$ variables via eq.(3.14) we finally have

$$
Z_{S t o c h}^{C S}=\int D A_{\mu} D b D \chi_{\alpha \beta} D \psi_{\mu} D c D \rho D \lambda D \phi e^{-S_{e f f}} .
$$

Here $\lambda$ and $\phi$ are (Grassman even) ghosts (with ghost number -2 and 2 respectively), introduced in order to exponentiate the determinant associated with the change from $\beta$ to $c$ variables. Concerning $\chi_{\alpha \beta}$, we have traded the three components of ghost field $\chi_{i}$ for those of a self-dual antisymmetric 
ghost (again with ghost number -1 ). This is done to make contact with the usual notation adopted in the literature [1, 9, 10], and, moreover, to take into account the correct tensorial character of this field [11] The effective action $S_{\text {eff }}$ reads:

$$
\begin{aligned}
S_{e f f}=\int_{M_{4}} & \operatorname{tr}\left\{\frac{1}{2}\left(F_{0 i}^{+}-\frac{4 \pi}{\kappa \Lambda^{2}} D_{j} F_{j i}\right)^{2}+\frac{1}{2}\left(\partial_{\mu} A_{\mu}\right)^{2}+\rho\left(D_{\mu} D_{\mu} c-D_{\mu} \psi_{\mu}\right)+\right. \\
& +\frac{4 \pi}{\kappa \Lambda^{2}} \chi_{0 i}\left(\delta_{i j} D_{k} D_{k}-D_{i} D_{j}\right) \psi_{j}-\chi_{\mu \nu} D_{[\mu} \psi_{\nu]}-b \partial_{\mu} \psi_{\mu}+ \\
& \left.+\lambda D_{\mu} D_{\mu} \phi+\lambda\left(\left[\psi_{\mu}, \psi_{\mu}+D_{\mu} c\right]+D_{\mu}\left[\psi_{\mu}, c\right]\right)\right\} d^{4} x
\end{aligned}
$$

In order to write $S_{\text {eff }}$ in a more tractable way, let us introduce Lagrange multipliers $\eta_{\alpha \beta}$ (self-dual and antisymmetric) and $\eta$, and the following (BRST) transformations:

$$
\begin{array}{ll}
\left\{Q, A_{\mu}\right\}=\psi_{\mu} & \left\{Q, \psi_{\mu}\right\}=0 \\
\{Q, b\}=\eta & \{Q, \eta\}=0 \\
\left\{Q, \chi_{\alpha \beta}\right\}=\eta_{\alpha \beta} & \left\{Q, \eta_{\alpha \beta}\right\}=0 \\
\{Q, c\}=\phi & \{Q, \phi\}=0 \\
\{Q, \lambda\}=\rho & \{Q, \rho\}=0
\end{array}
$$

Then, $S_{\text {eff }}$ can be written in the form:

$$
S_{e f f}=\left\{Q, V_{\Lambda}\right\}
$$

with

$$
\begin{gathered}
V_{\Lambda}=\int_{M_{4}} d^{4} x \operatorname{tr}\left\{\frac{1}{4} \chi_{\alpha \beta}\left(F_{\alpha \beta}^{+}-\frac{1}{2} \eta_{\alpha \beta}\right)-\chi_{0 i} \frac{4 \pi}{\kappa \Lambda^{2}} D_{j} F_{j i}+\right. \\
\left.+b\left(\partial_{\mu} A_{\mu}-\frac{1}{2} \eta\right)-\lambda\left(D_{\mu} \psi_{\mu}+D_{\mu} D_{\mu} c\right)\right\}
\end{gathered}
$$

In this way, we can easily see that the $\Lambda$-independent part of $S_{\text {eff }}$ coincides with the action constructed by Witten for defining TYM theory [1]]. Accordingly, the BRST transformations (3.18) correspond to those introduced by Labastida and Pernici [5] in order to obtain TYM as the result of gauge fixing a gaussian trivial action. To see this correspondence explicitly, one has to proceed to the following change of variables [30] :

$$
\begin{aligned}
\psi_{\mu} & \rightarrow \psi_{\mu}+D_{\mu} c \\
\phi & \rightarrow \phi+\frac{1}{2}[c, c] .
\end{aligned}
$$


Then, transformations (3.18) coincide with the BRST transformations defined in Ref. [5] in order to handle the large topological symmetry characteristic of TYM theory.

We can now write the following equality which turns to be one of the main results in our work:

$$
Z_{S t o c h}^{C S}[\Lambda]=Z_{B R S T}^{T Y M}[\Lambda]
$$

where

$$
Z_{B R S T}^{T Y M}[\Lambda]=\int D \Phi e^{\left\{Q, V_{\Lambda}\right\}}
$$

and $D \Phi$ represents the integral measure over the whole field content. Hence, the stochastic partition function for the $d=3$ CS model regulated with a Yang-Mills term coincides with the BRST partition function for a $d=4$ TYM model with the addition of a $\Lambda$-dependent functional (eq.(3.20)).

The fact that the $\Lambda$-dependent terms in $S_{\text {eff }}$ appear through $Q$-exact terms allows us to proceed one step further. Indeed, since

$$
\frac{\delta Z_{B R S T}^{T Y M}[\Lambda]}{\delta \Lambda}=-\int D \Phi\left\{Q, \frac{\partial V_{\Lambda}}{\partial \Lambda}\right\} e^{\left\{Q, V_{\Lambda}\right\}},
$$

then

$$
\frac{1}{Z} \frac{\left.\left.\delta Z_{B R S T}^{T Y M}\right] \Lambda\right]}{\delta \Lambda}=-<\left\{Q, \frac{\partial V_{\Lambda}}{\partial \Lambda}\right\}>=0
$$

due to the vanishing of v.e.v.'s of BRST commutators [1]. Then, at the partition function level we infer that, being $Z_{B R S T}^{T Y M}[\Lambda] \Lambda$-independent, it can be evaluated in the limit $\Lambda$ going to infinity. Hence, we can write instead of (3.22)

$$
Z_{\text {Stoch }}^{C S}=Z_{B R S T}^{T Y M},
$$

where in the r.h.s one has now the partition function for TYM theory with the action constructed by Witten. This does not mean that from the stochastic point of view the regulator is not necessary. As explained in the previous Section, in order to compute CS propagators, etc., one has to mantain the regulator till the end of the computations. Eq.(3.26) implies that one can use a regulator $\Lambda$ to render all quantities appearing in the derivation of (3.22) well-defined; at the end of the computations, being the resulting partition function $\Lambda$-independent, one can make $\Lambda \rightarrow \infty$ thus confirming the connection between CS and TYM models. In this respect, one can think of identity 
(3.26) as follows: from the CS point of view one has to mantain the regulator in order to have a convergent stochastic process, yielding the correct stochastic averages. One then makes $\Lambda \rightarrow \infty$ at the end of the computations. From the TYM point of view, due to (3.25) one can make $\Lambda \rightarrow \infty$ at any step of the computations, not only for $Z_{B R S T}^{T Y M}$ but for any v.e.v. to be computed.

Indeed, the basic formula (3.19) also ensures that

$$
<T_{\mu \nu}>=-\frac{2}{\sqrt{g}} \frac{\delta \log Z_{B R S T}^{T Y M}[\Lambda]}{\delta g^{\mu \nu}}=0 .
$$

That is, $Z_{B R S T}^{T Y M}$ defines a topological model for any value of $\Lambda$. As we discussed above, in order to prove (3.27) one should use Fujikawa variables but, when written in these terms, one can still prove that $S_{\text {eff }}$ is a $Q$-exact form. We skip details since they parallel those presented in Ref. [11].

Analogously, one has, for arbitrary $\Lambda$

$$
\frac{\partial Z_{B R S T}^{T Y M}[\Lambda]}{\partial e^{2}}=0 .
$$

The independence of the partition function for TQFT's on the coupling constant has as an important consequence that one can compute it in the weakcoupling (semi-classical) limit. On the one hand, for TYM theory (i.e. once the limit $\Lambda \rightarrow \infty$ is taken) one can therefore write $Z_{B R S T}^{T Y M}$ as a sum over contributions from the neighbourhoods of the isolated instantons since classical minima of the TYM action correspond to solutions of

$$
F_{\mu \nu}^{+}=0 .
$$

In expanding around isolated instantons it is then enough to keep only quadratic terms and the resulting gaussian integrals give:

$$
\frac{\text { Pfaff } D_{F}}{\sqrt{\operatorname{det} \Delta_{B}}},
$$

where Pfaff $D_{F}$ denotes the Pfaffian of the operator associated to Fermi fields and $\Delta_{B}$ that associated to Bose fields in the quadratic part of the action. Up to a sign, this ratio is 1 and then

$$
Z_{B R S T}^{T Y M}=\sum_{\text {instantons }}( \pm 1),
$$


that is, it coincides with the first Donaldson invariant.

On the other hand, for $Z_{B R S T}^{T Y M}[\Lambda]$ at finite $\Lambda$, classical minima for action $S_{\text {eff }}$ are not any more solutions of (3.29), although in the weak-coupling limit gaussian integrals give a ratio like (3.30) which is still \pm 1 . However, the partition function will yield the first Donaldson invariant provided one takes the $\Lambda \rightarrow \infty$ limit.

It is important to notice that the $\Lambda$-independence of $Z_{B R S T}^{T Y M}$ extends to any (topological invariant) observable. Indeed, the sufficient conditions for an observable $\Theta$ to give a topologically invariant v.e.v. are [1]:

1) $\delta \Theta / \delta g^{\mu \nu}=\{Q, R\}$

2) $\{Q, \Theta\}=0$ (modulo those of the form $\Theta=\{Q, B\}$ ).

Then, being $\Theta \Lambda$-independent, one has

$$
\begin{aligned}
\frac{\partial<\Theta>}{\partial \Lambda} & =-\int D \Phi e^{-S_{\text {eff }}\left\{Q, \frac{\partial V}{\partial \Lambda}\right\} \Theta} \\
& =-<\left\{Q, \frac{\partial V}{\partial \Lambda} \Theta\right\}>=0 .
\end{aligned}
$$

At this point it is interesting to make contact with the results of Chen et al. 24] on topological features in perturbative CS theory. Using both dimensional and $\operatorname{tr} F_{i j}^{2}$ regularizations, these authors showed the vanishing of the $\beta$ function for CS theory up to three loops and also suggested the absence of diffeomorphism anomaly (which could be present due to the metric dependence of the $\operatorname{tr} F_{i j}^{2}$ regulator). Indeed, by studying the propagator for the regulated CS model they showed that its metric dependent transverse part (responsible for the would-be diffeomorphism anomaly) vanishes because of the unusual properties of the wave-function renormalization. Our results, summarized by eqs. (3.22) and (3.25) confirm this suggestion.

Concerning the evaluation of topological invariants using the connection between CS and TYM theories, let us note that the modifications of the SQ procedure needed whenever $\operatorname{dim} \mathcal{M} \neq 0$ make the analysis more involved [9]. However, the conclusions of Ref.[9] about the connection between topological invariants of TYM theory and those arising in $d=3$ CS theory should continue to hold when the regulation of the stochastic process is appropriately taken into account.

We shall conclude this Section discussing an alternative to the use of a $\frac{1}{\Lambda^{2}} \operatorname{tr} F_{i j}^{2}$ regulator, namely, the introduction of a non-trivial kernel $K_{i j}$ in the Langevin equation in order to have a stochastic process which converges to 
equilibrium. In fact, this alternative approach was followed in Refs. 21, 22] in the study of the Abelian and non-Abelian Chern-Simons models. In this last case, if one chooses $K_{i j}$ in the form:

$$
K_{i j}=-\epsilon_{i j k} \partial_{k},
$$

then the generalized Langevin equation:

$$
\dot{A}_{i}^{a}=K_{i j} \frac{\delta S}{\delta A_{j}^{a}}+\eta_{i}^{a},
$$

yields the correct equilibrium limit to all orders in perturbation theory so that conventional propagators are nicely obtained [21].

Now, if starting from eq.(3.34) one tries to repeat the steps leading to the identification of the stochastic partition function associated with the CS model and the BRST partition function for the TYM theory (eq.(3.22)), there are difficulties originated in the appearence of non-local terms, related to the presence of kernel $K_{i j}$.

There is however an alternative derivation of the CS-TYM connection presented in Ref.[7]. As we shall see, it is easy to prove, using this approach, the desired connection in the presence of a kernel. To this end, let us rewrite Langevin equation (3.34) and the corresponding Chern-Simons action in the form:

$$
\begin{gathered}
\dot{A}=K \frac{\delta S_{C S}}{\delta A}+\eta, \\
S_{C S}=-\kappa \pi \int_{M_{3}} d^{3} x \mathcal{K}_{0}(x),
\end{gathered}
$$

where for simplicity we have avoided indices and introduced the notation $\mathcal{K}_{0}$ for the CS Lagrangian for later convenience (compare eq.(2.2)). The stochastic partition function associated with the generalized Langevin equation (3.34) reads:

$$
Z_{S t o c h}\left[S_{C S}\right]=\int D \eta \exp \left[-\int d^{3} x d^{3} y d t \eta(x, t) K^{-1}(x, y) \eta(y, t)\right]
$$

with $K^{-1}(x, y)$ the inverse of kernel $K$ (in fact, one has to slightly modify $K_{i j}$ in order to make it inversible by adding a longitudinal part, $K_{i j} \rightarrow K_{i j}=$ 
$-\epsilon_{i j k} \partial_{k}+\alpha \partial_{i} \partial_{j}$, and treating $\alpha$ as a gauge fixing parameter). Now, using eq. 3.34) we can rewrite (3.37) in the form:

$$
\begin{aligned}
Z_{\text {Stoch }}\left[S_{C S}\right] & =\int D A \operatorname{det}\left(\frac{\partial}{\partial t}-K \frac{\delta^{2} S_{C S}}{\delta A^{2}}\right) \times \\
& \times \exp \left[-\operatorname{tr} \int d^{3} x d^{3} y d t\left(\dot{A} K^{-1} \dot{A}-2 \dot{A} \frac{\delta S_{C S}}{\delta A}\right.\right. \\
& \left.\left.+K\left(\frac{\delta S_{C S}}{\delta A}\right)^{2}\right)\right] .
\end{aligned}
$$

Consider now the $(3+1)$-dimensional action:

$$
S^{(3+1)}=\operatorname{tr} \int_{M_{4}} d^{3} x d t \frac{\delta S_{C S}}{\delta A} \dot{A}
$$

where gauge fields depend now on an additional (fictitious) time $t, t \in I \equiv$ $[0, T]$ (at the end of the computations one should let $T \rightarrow \infty$ ). It is easy to verify that action (3.39) is invariant under the following (large) transformation:

$$
\delta A=\delta \epsilon(x, t)
$$

provided

$$
\delta \epsilon(x, 0)=\delta \epsilon(x, T)=0
$$

Let us use the standard BRST procedure in order to fix this large invariance. The corresponding partition function reads:

$$
Z_{B R S T}\left[S^{(3+1)}\right]=\int D A D b D \chi D \psi \exp \left[-S^{(3+1)}-\operatorname{tr} \int_{M_{4}} d^{3} x d t\left\{Q, \chi\left(F-\frac{b}{2}\right)\right\}\right]
$$

with $b$ a Lagrange multiplier enforcing the gauge condition (which at the end will turn to be $F=0$ ) and $\chi$ and $\psi$ the ghost fields (which in our previous derivation were introduced through eqs.(3.13)). BRST transformations $\{Q, \quad\}$ are defined as:

$$
\begin{array}{ll}
\{Q, A\}=\psi & \{Q, b\}=0 \\
\{Q, \chi\}=K^{-1} b & \{Q, \psi\}=0
\end{array}
$$


With this, we have:

$$
\begin{aligned}
Z_{B R S T}\left[S^{(3+1)}\right] & =\operatorname{tr} \int D A D b D \chi D \psi \times \\
& \times \exp \left[-S^{(3+1)}-\int_{M_{4}} d^{3} x d t\left(K^{-1} b\left(F-\frac{b}{2}\right)+\chi \frac{\delta F}{\delta A} \psi\right)\right]
\end{aligned}
$$

or, integrating out $b$ :

$$
Z_{B R S T}\left[S^{(3+1)}\right]=\int D A D \chi D \psi \exp \left[-S^{(3+1)}-\operatorname{tr} \int_{M_{4}} d^{3} x d t\left(\chi \frac{\delta F}{\delta A} \psi+\frac{1}{2} K^{-1} F^{2}\right)\right]
$$

Then, if we choose as gauge function

$$
F=\dot{A}-K \frac{\delta S_{C S}}{\delta A}
$$

we get:

$$
\begin{aligned}
& Z_{B R S T}\left[S^{(3+1)}\right]=\int D \operatorname{Adet}\left(\frac{\partial}{\partial t}-K \frac{\delta^{2} S_{C S}}{\delta A^{2}}\right) \times \\
& \quad \times \exp \left[-\operatorname{tr} \int_{M_{4}} d^{3} x d t\left(\dot{A} K^{-1} \dot{A}-2 \dot{A} \frac{\delta S_{C S}}{\delta A}+K\left(\frac{\delta S_{C S}}{\delta A}\right)^{2}\right)\right.
\end{aligned}
$$

and comparing with eq. (3.38) we have:

$$
Z_{B R S T}\left[S^{(3+1)}\right]=Z_{S t o c h}\left[S_{C S}\right]
$$

It remains to show that $S^{(3+1)}$ is indeed the classical action associated with TYM theory. To this end note that:

$$
S^{(3+1)}=Q[T]-Q[0]
$$

where:

$$
Q(t) \equiv-\kappa \pi \int_{M_{3}} d^{3} x \mathcal{K}_{0}
$$

Now, if we define a gauge field $A_{\mu}$ in $(3+1)$-dimensions (in the $A_{0}=0$ gauge) as $A_{\mu}=\left(A_{i}, 0\right)$, one can easily prove [31] that: 


$$
\lim _{T \rightarrow \infty} Q[T]=-\kappa \pi \int_{M_{4}} d^{4} x \partial_{\mu} \mathcal{K}_{\mu}
$$

where $\mathcal{K}_{\mu}$ is the Chern-Simons characteristic class:

$$
\mathcal{K}_{\mu}=-\frac{1}{16 \pi} \operatorname{tr} \epsilon_{\mu \nu \alpha \beta}\left(F_{\nu \alpha} A_{\beta}-\frac{2}{3} A_{\nu} A_{\alpha} A_{\beta}\right)
$$

(One has to choose initial conditions $A_{i}(x, 0)=0$ so that $Q[0]=0$ ). One then has, after taking the $T \rightarrow \infty$ limit:

$$
S^{(3+1)}=-\kappa \pi \int_{M_{4}} d^{4} x \partial_{\mu} \mathcal{K}_{\mu}
$$

or

$$
S^{(3+1)}=\frac{\kappa}{16 \pi} \operatorname{tr} \int_{M_{4}} d^{4} x^{*} F_{\mu \nu} F_{\mu \nu}
$$

That is, action $S^{(3+1)}$ coincides with the Chern-Pontryagin invariant which was taken by Baulieu and Singer [33] as starting classical action to obtain, after BRST quantization, Witten's TYM theory. Then, eq.(3.48) states the equivalence between the stochastic partition function for CS theory with kernel $K$ and the partition function for TYM theory (constructed after quantization of classical action (3.54)).

A comment is here in order: we have not introduced in our derivation neither ordinary ghosts associated with ordinary gauge invariance nor ghost for ghost, necessary to fix the second generation gauge-invariance characteristic of TYM theory. We have ignored them just to present more clearly our arguments, but these ghosts can be easily included following the same steps leading from eq.(3.8) to eq.(3.17) and our proof remains still valid. In any case, we have shown that the equivalence between TYM and CS partition functions can be also proven when a non-trivial kernel is introduced in the Langevin equation in order to handle the convergence to equilibrium problem which arises for CS theory. The interesting fact is that in this approach convergence can be proven to all orders in perturbation theory [21].

\section{Conclusions}

In the last few years the interest in Topological Field Theories has prompted cross-fertilizing investigations both in Mathematics and in Physics. One 
important result of these studies is that they allow to establish multiple connections between field-theoretical models in different number of spacetime dimensions.

One example of this is the connection discovered by Witten [2] between $d=3$ Chern-Simons gauge theory and $d=2$ rational conformal field theories: the states in the Hilbert space of the CS theory defined on a compact surface is the space of conformal blocks of rational conformal field theories. Another example is the relation between $d=3$ CS theory and $d=4$ Topological YangMills theory [9, 10]: topological invariants in TYM theory correspond to observables in CS theory.

These connections have been established using different approaches: pathintegral quantization, canonical quantization, stochastic quantization. This last approach is the one we have employed in the present work to re-analyze the relation between $d=3$ CS and $d=4$ TYM theories (although other relations like for example that connecting $d=1$ spin model and $d=2$ topological sigma model 35] can be studied using an identical approach).

The original studies [9]-11] of the CS-TYM connection using SQ were somehow formal in the sense that the non-convergence of the underlying stochastic process was disregarded. Later on, the problem of convergence in SQ of CS theory was solved [21, 22] by introducing appropriate kernels or regulators so that a correct equilibrium limit can be attained. After these results, it remained to determine whether the connection between CS and TYM theories was still valid in the presence of regulators. One of the aims of the present work was to address to this question and the answer is affirmative. As we have shown in Section 3, the addition of a regulator $\frac{1}{\Lambda^{2}} \operatorname{tr} F_{i j}^{2}$ to the CS action does not affect the proof of equivalence between the stochastic partition function for CS theory and the BRST partition function for TYM theory.

Indeed, due to the fact that the effective 4-dimensional action $S_{\text {eff }}$ is a $Q$-exact form even in the presence of a regulator $\Lambda$ (see eq.(3.19)), the resulting partition function $Z_{B R S T}^{T Y M}[\Lambda]$ is independent of $\Lambda$. Hence, it can be evaluated in the limit $\Lambda \rightarrow \infty$ where it coincides with the TYM partition function defined by Witten [1]. In any case, for arbitrary $\Lambda, Z_{B R S T}^{T Y M}[\Lambda]$ defines a topological theory, since the corresponding v.e.v. for the energy-momentum tensor vanishes (see eq.(3.27)) and all topologically invariant observables are $\Lambda$-independent (see eq. (3.29)).

In the course of this work we have also found of interest to study, in 
the same vein as Namiki et al. [26] did for the SQ of Yang-Mills theory, how convergence for non-Abelian CS model is attained when the regulator is introduced and the precise way in which standard propagators are obtained using SQ. We have proven that the addition of a regulator $\frac{1}{\Lambda^{2}} \operatorname{tr} F_{i j}^{2}$ to the CS action renders the stochastic process associated with the CS Langevin equation convergent. The proof we have presented is valid up to second order in perturbation theory and states that the transverse part of the stochastic diagrams reproduces the standard gluonic CS propagator (see eq.(2.24)). It is interesting to note that the way in wich stochastic diagrams for CS regulated theory sum up to the standard propagator is basically identical to the way it works in the case of pure YM theory.

We have also discussed the alternative regularization scheme which consists in the introduction of a non-trivial kernel. Indeed, an appropriate choice of the kernel [21, 22] (see eq.(3.33)) yields the correct equilibrium limit to all orders in perturbation theory. As we have shown, also in this approach it becomes clear that the equivalence between the stochastic partition function for CS theory and the BRST partition function for TYM theory can be proved in the presence of a regulator (in this case a kernel). 


\section{TABLE CAPTIONS.}

Table 1: Diagrams for stochastic vertices.

FIGURE CAPTIONS.

Figure 1: Perturbative expansion of $A_{i}^{a}(k, t)$.

Figure 2: Graphical representation of $\mathcal{D}_{i j}^{a b}(k, t)$. 


\section{References}

[1] E.Witten, Comm.Math.Phys. 117 (1988) 353.

[2] E.Witten, Comm.Math.Phys.118 (1989) 411.

[3] M.Dune, R.Jackiw and M.Trugenberger, Ann. of Phys.(N.Y.) 194 (1989) 197.

[4] M.Blau, D.Birmingham, M.Rakowski and G.Thompson, Phys.Rep. $\mathbf{2 0 9}(1991) 129$.

[5] J.Labastida and Pernici, Phys.Lett. 212B (1988) 91.

[6] D.Birmingham, M.Rakowski and G.Thompson, Phys.Lett. 212B (1988) 187; ibid 214B (1988) 381.

[7] G.Horowitz, Comm.Math.Phys. 125 (1989) 417.

[8] L.Baulieu and B.Grossmann, Phys.Lett. 212B (1988) 351.

[9] Y.Yu, Phys.Rev. D40 (1989) 1301.

[10] L.Baulieu, Phys.Lett. 232B (1989)479.

[11] L.Cugliandolo, G.Lozano and F.A.Schaposnik, Phys.Lett. 253B (1991) 90.

[12] G.Parisi and Y.S.Wu, Sci.Sinica 24 (1981) 483.

[13] G.Parisi and N.Sourlas, Nucl.Phys. B206 (1982) 321.

[14] S.Cecotti and L.Girardello, Ann. of Phys.(N.Y.) 145 (1983) 81.

[15] K.Fujikawa, Phys.Rev.Lett. 42 (1972) 1195; ibid 44 (1980) 1733; Phys.Rev.D38 (1980) 2848.

[16] L.Cugliandolo, G.Lozano and F.A.Schaposnik, Phys.Lett. 244B (1990) 249.

[17] R.K.Kaul and R.Rajaraman, Phys.Lett. 249B (1990) 433.

[18] G.Parisi, Phys.Lett. 131B (1983) 393. 
[19] J.Ambjorn and S.K.Yang, Phys.Lett. 165B (1985) 140.

[20] A.Polykronakos and R.Tzani, Phys.Lett. 259B (1991) 291.

[21] Y.S.Wu and Zhu, Univ. of Utah preprint, 1991.

[22] F.Ferrari and H.Huffel, Phys.Lett. 261B (1991) 47.

[23] R.Jackiw and S.Templeton, Phys.Rev. D23 (1981) 2291;

S.Deser, R.Jackiw and S.Templeton, Phys.Rev.Lett. 49 (1982)975; Ann.Phys 140 (1982) 372.

[24] W.Chen, G.W.Semenoff and Y.S.Wu, Mod.Phys.Lett.A23 (1990) 1833.

[25] D.Zwanziger, Nucl.Phys.B192 (1981) 259.

[26] M.Namiki, I.Ohba, K.Okano and Y.Yamanaka, Prog.Theor.Phys. 69 (1983) 1580.

[27] P.H.Damgaard and H Hüffel, Phys.Rep. 152 (1987) 227.

[28] R.Pisarski and S.Rao, Phys.Rev. D22 (1985) 2081.

[29] Alvarez-Gaume, Labastida and Ramallo, Nucl.Phys. B334 (1990) 103

[30] S.Ouvry, R.Stora and P.van Baal, Phys.Lett. 220B (1989) 159.

[31] See e.g. R.Jackiw in Topological Investigations of Quantized Gauge Theory, Les Houches Session XL, 1983, eds. B.S. de Witt and R.Stora, Elsevier Science Pub. B.V., 1984.

[32] L.Baulieu, Phys.Lett. B167 (1986) 421; Nucl.Phys. B270 (1986) 507.

[33] L.Baulieu and I.M.Singer, Nucl.Phys. B5 (Proc.Suppl.) (1988) 142.

[34] L.Cugliandolo, G.Rossini and F.A.Schaposnik, Phys.Lett. 267B (1991) 200.

[35] E.Fradkin, E.Moreno and F.A.Schaposnik, Phys.Rev. D, in press (1991). 